\title{
Cerebral thromboangiitis associated with Covid-19 infection: a young woman case report
}

\begin{abstract}
COVID-19 may predispose to both venous and arterial thromboembolic disease due to excessive inflammation, hypoxia, and immobilization. Remarkably, thrombotic complications have hardly been described. We describe a patient with severe pneumonia, COVID-19 correlated, who developed a presumable and rare form of thromboangioiitis with cerebrovascular thrombotic complication and neurological symptoms, in absence of other cardio-vascular risk factor. Significant inflammation is present in our patient, which may explain more dramatic activation of coagulation and arterial endothelium vessels damage. The probable mechanism of this cardiovascular injury may be a direct effect of coronavirus related to Angiotensin-converting enzyme 2 receptor (ACE2-r). The exact mechanisms by which COVID-19 induced hypercoagulability remain incompletely elucidated and more research are necessary.
\end{abstract}

Volume I 3 Issue I - 202 I

\author{
Mirabella Lucia,' Barberio Francesco,' \\ Caporusso Roberta,' Tullo Livio,' Labella \\ Daniela,' Mariano Karim,' Rauseo Michela,' \\ Macarini Luca, ${ }^{2}$ Cinnella Gilda' \\ 'Department of Intensive Care, University of Foggia, Italy \\ 2Department of Radiology and Radioterapy, University of Foggia, \\ Italy
}

Correspondence: Mirabella Lucia, Department of Intensive
Care, University of Foggia, Italy, Tel +3934077 I 221 I, Fax 088I732387,Email Lucia.mirabella@unifg.it

\section{Introduction}

The novel Coronavirus (COVID-19) can cause severe pneumonia and respiratory symptoms that need admission to Intensive Care Unit for respiratory support. In many cases, systemic thrombotic complications have been described, including venous thromboembolism and arterial thrombotic events. ${ }^{1}$ Reports on the incidence of thrombotic complications are however not available but focus on arterial embolism, Covid-19 related, is important for decision making concerning thromboprophylaxis, especially in patients admitted to the intensive care unit (ICU) who are at highest thrombotic risk. We describe a young patient with a severe form of COVID-19 who developed cerebrovascular thrombotic complications with neurological symptoms and cerebral vascular disease suggestive for viral thromboangiitis, in absence of other cardio-vascular risk factors or another cardio-vascular risk factor.

\section{Case report}

A 29-year-old woman was admitted to the Intensive Care Unit for interstitial pneumonia with respiratory distress in March 2020, after 7 days of cough and fever. The patient's medical history was relevant for not specified psychiatric disease treated with Sodium Valproate and Sertraline. Physical examination revealed a body temperature of $37,4^{\circ}$, blood pressure $130 / 85 \mathrm{mmHg}$, pulse 115 beats per minute, respiratory rate of 60 breaths per minute, with oxygen saturation $\left(\mathrm{SpO}_{2}\right)$ 65\% room air, rhonchi at auscultation. Reverse transcriptasepolymerase chain reaction assay of a nasopharyngeal swab tested positive for severe acute respiratory syndrome coronavirus-2 (SARSCoV-2). Chest radiography was performed, which reported patchy air space opacification in both lung middle-lower zones with diffuse ground-glass haze bilaterally, results consistent with pneumonia (Figure 1).

In accordance with hospital guidance, infection was treated with a combination of antiretroviral drugs (Darunavir/Cobicistat) and Hydroxychloroquine, then Enoxaparin 6000UI twice a day was introduced. 12 days later, the patient experienced an improvement of the pulmonary disease as shown at chest radiography and blood gas analysis $(\mathrm{P} / \mathrm{F}$ ratio $=220)$, the pharmacological coma was suspended. The patient was contactable, carried out simple orders but the neurological examination revealed left-side hemiplegia. A noncontrast head CT scan was immediately performed, which a large area of hypo-attenuation with loss of gray-white matter differentiation involving the right frontal and parietal lobes, the lentiform nucleus, and the caudate head, with effacement of sulci; findings are indeterminate and appearance in keeping with hypoxic-ischemic injury (Figure 2). On day 14 a Magnetic Resonance Imaging (MRI) scan was performed, which reported "large right cortical and subcortical lesion of an altered signal involving the vascular distribution territory of the Anterior Cerebral Artery (ACA) and the upper and deep cortical areas of the Middle Cerebral Artery (MCA), with signs of hemorrhagic infarction, cortical laminar necrosis and wallerian degeneration of cortical-spinal tract. The distribution and morphology of subacute hypoxic-ischemic lesions and the angio-MRI findings, in the absence of cardiovascular risk factors, were supposed to be suggestive of viral thromboangiitis (Figure 3).

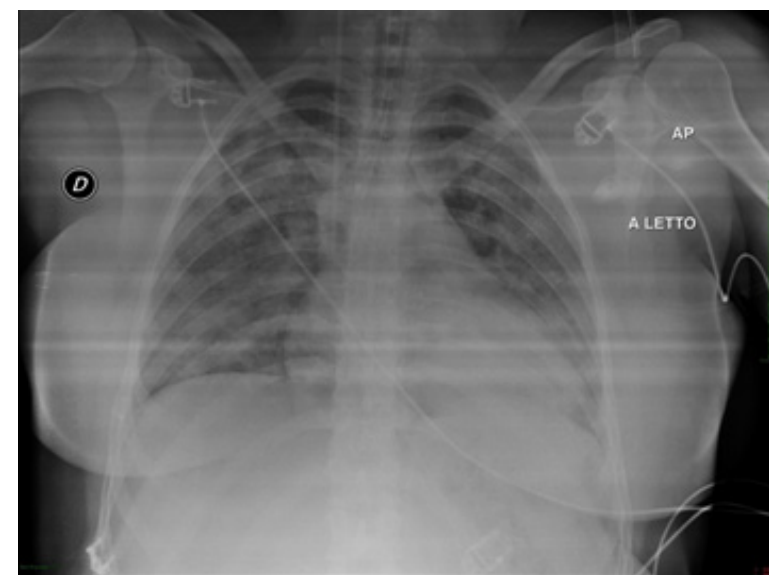

Figure I Anteroposterior Chest Radiographs March 2020. Patchy air space opacification in both lung middle-lower zones with diffuse ground glass haze bilaterally, results consistent with pneumonia.

During the weaning phase, the patient presented hypertension resistant at medications. Screening for thrombophilia, pheochromocytoma, malignant hypertension, common serum autoantibody (anti-CCP antibodies, antinuclear antibodies (ANAs), anti-neutrophil cytoplasmic antibodies (ANCA) and anti-phospholipid 
antibodies) resulted negative. On the results of the MRI, corticosteroid therapy and physio-kinetic therapy were set up. The patient gradually resumed mobility on the left leg and partially on the left arm. On hospital day 60, pulmonary disease solved, with nasopharyngeal swabs tested negative for $2019-\mathrm{nCoV}$, the patient was transferred to the rehabilitation unit.

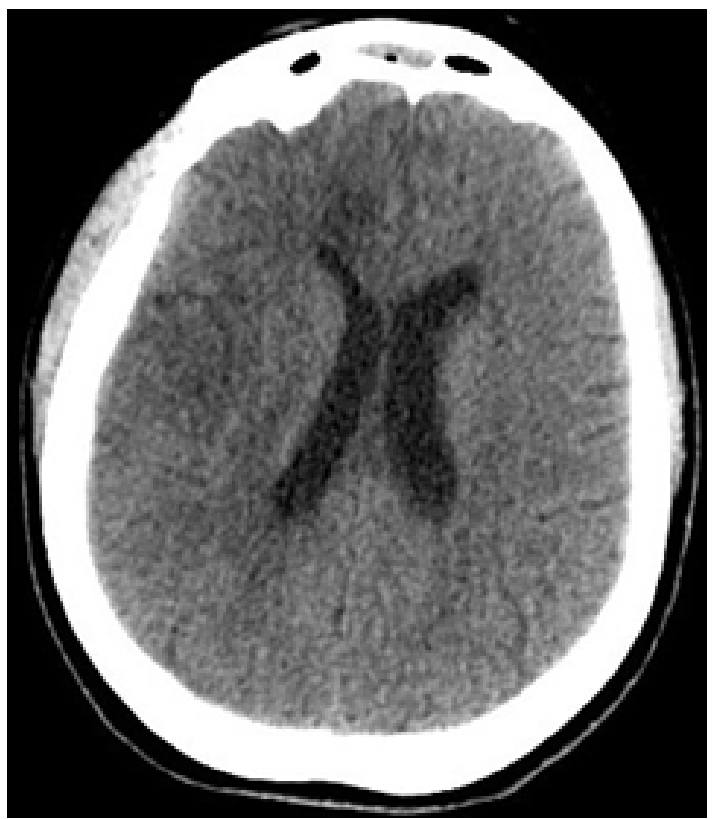

Figure 2 Non-contrast head CT demonstrate large area of hypo-attenuation with loss of gray matter differentiation involving the right frontal and parietal lobes, the lentiform nucleus and the caudate head, with effacement of the sulci.

\section{Discussion}

Our report illustrates a rare manifestation of cerebral viral thromboangiitis in a young woman affected by COVID-19 pneumonia in absence of other thrombotic risk factors. Several studies described ischemic and neurological symptoms in patients affected by Severe Acute Respiratory Syndrome (SARS) in 2003 and during COVID 19 outbreak. ${ }^{2,3}$ According to literature COVID 19 may predispose to, both venous and arterial, thromboembolic complications caused by an inflammatory response, with increasing of inflammatory cytokines, D-dimers, and leukocytes levels, which leads to coagulopathy. ${ }^{4}$ The probable mechanism of this cardiovascular injury may be related to Angiotensin-converting enzyme 2 receptor (ACE2-r) that has been identified as a functional receptor for coronaviruses. ${ }^{5}$ Through ACE2-r, COVID-19 invades not only alveolar epithelial cells, resulting in pneumonia but also the endothelium of the cardiovascular system, inducing vascular inflammation and thromboembolic complications. As reported, significant inflammation is present in patients with $2019 \mathrm{nCoV}-2$ infection, with elevated levels of IL-6, $\mathrm{C}$-reactive protein, and D-dimers, and the subsequent activation of coagulation. . $^{1,6}$ Our patient, as others, described, should have had a more pronounced inflammatory response to infection with SARS-

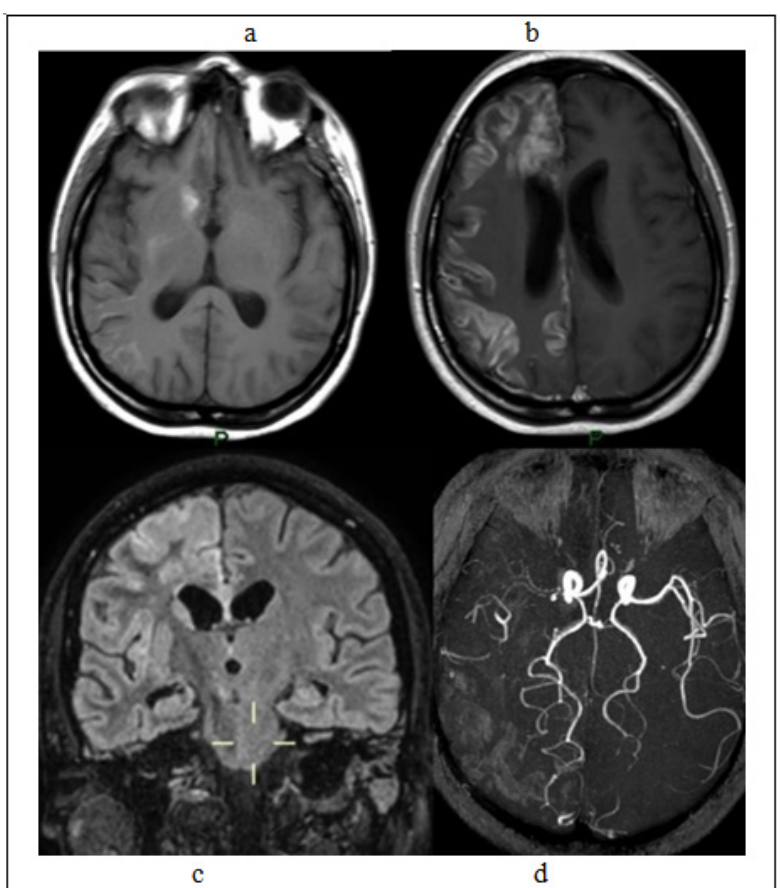

Figure $3 \mathrm{MRI}$ images demonstrate hemorrhagic infarction of lentiform nucleus and the caudate head and cortical laminar necrosis parietal on TI SE (a) gyroscopic enhancement frontal and parietal on TI SE after the contrast (b), cortical and subcortical lesion frontal-parietal and Wallerian degeneration of the corticospinal tract on FLAIR(c); azygos ACA and stenosis of MI before the early bifurcation with a filiform and irregular appearance of two trunks on.

CoV-2, which may explain more dramatic changes in coagulation and arterial endothelium vessels.

As shown in Table 1, levels of pro-inflammatory values in our patient increase in the second week and could be compatible with the ischemic cerebral damage observed and start to decrease once corticosteroid therapy was started. Despite the link between ACE2-r and Coronavirus mechanism could explain the vascular implication observed in $2019 \mathrm{nCoV}-2$ patients, other mechanisms should be investigated to better define this arteritis cause. Arteritis pathogenesis could be "primarily" or "secondary". In "primarily" arteritis inflammation of wall vessels is caused by an excess of autoantibodies directed against the endothelium; in many cases, the etiology of these autoantibodies is unknown. "Secondary" arteritis are due to dysregulated immune response to infections, cancer or other autoimmune diseases. We can speculate that COVID-19 disease stimulating immune system lead to a production of cross-antibodies against wall vessels causing observed arteritis. In this case, the search for the common autoantibody panel had negative results, therefore the underlying mechanism that could have determined the onset of arteritis, could be ascribed to other, not yet defined antibody reaction of the COVID-19 disease.

Table I Timeline of infiammatory values

\begin{tabular}{lllllll}
\hline ICU Day & D-Dimers & Procalcitonin & C-Reactive Protein & Creatine Phosphokinase & Myoglobin & WBC \\
\cline { 2 - 8 } & $\mathbf{n g} / \mathbf{m L}$ & $\mathbf{n g} / \mathbf{m L}$ & $\mathbf{m g} / \mathbf{L}$ & $\mathbf{U} / \mathbf{L}$ & $\mathbf{n g} / \mathbf{m L}$ & $\mathbf{1 0} \wedge^{3} / \mathbf{u L}$ \\
\hline I & 6.912 & 3,62 & - & 75 & 42,1 & 14790 \\
3 & 6.520 & 1,53 & 258,90 & 32 & 34,6 & 12980 \\
7 & 21.370 & 0,03 & - & 250 & 122,00 & 14200 \\
\hline
\end{tabular}


Table continued...

\begin{tabular}{|c|c|c|c|c|c|c|}
\hline \multirow[t]{2}{*}{ ICU Day } & D-Dimers & Procalcitonin & C-Reactive Protein & Creatine Phosphokinase & Myoglobin & WBC \\
\hline & $\mathrm{ng} / \mathrm{mL}$ & ng/mL & mg/L & U/L & ng/mL & $10 \wedge^{3} / \mathrm{uL}$ \\
\hline 9 & 19.500 & 0,03 & - & 83 & 98,70 & 12400 \\
\hline 10 & 14.039 & 0,30 & - & - & 102,90 & 10880 \\
\hline II & 13.392 & 0,03 & 322,50 & 508 & 70,70 & 14920 \\
\hline 12 & 7.304 & 0,02 & 216,70 & 29 & 92,10 & 14230 \\
\hline 13 & 7.090 & 0,45 & 116,50 & 32,00 & 109,10 & 10810 \\
\hline 19 & 4.961 & 0,03 & 0,55 & 63 & 91,50 & 9170 \\
\hline 20 & 6.777 & $<0,02$ & 9,10 & 39 & 69,50 & 9200 \\
\hline
\end{tabular}

\section{Conclusion}

Although the main manifestation of the COVID-19 infection is viral pneumonia, many different manifestations have been highlighted in other organs, especially regarding the cardiovascular system. Recent publications have highlighted a coagulation disorder in severely diseased patients, responsible for a hypercoagulable state which might explain disseminated intravascular coagulation and organ failure. Some hypothesize a direct effect of coronavirus on vascular endothelial cells to justify acute arterial thrombosis. Further mechanisms should be investigated to explain the arterial damage caused by COVID- 19 . ACE2-r pathway could explain cardiovascular involvement but on the basis on this patient imaging results autoimmune pathway should be investigated.

\section{References}

1. Klok FA, Kruip MJHA, van der Meer NJM, et al. Incidence of thrombotic complications in critically ill ICU patients with COVID-19. Thromb Res. 2020;191:145-147.
2. Mao L, Jin H, Wang M, Hu Y et al. Neurologic Manifestations of Hospitalized Patients With Coronavirus Disease 2019 inWuhan, China. JAMA Neurol. 2020;77(6):683-690.

3. Umapathi T, Kor AC, Venketasubramanian $\mathrm{N}$, et al. Large artery ischaemic stroke in severe acute respiratory syndrome (SARS). $J$ Neurol. 2004;251(10):1227-1231.

4. Connors JM, Levy JH. COVID-19 and its implications for thrombosis and anticoagulation. Blood. 2020;135(23):2033-2040.

5. Li H, Liu L, Zhang D et al. SARS-CoV-2 and viral sepsis: observations and hypotheses. Lancet. 2020;395(10235):1517-1520.

6. Zheng YY, Ma YT, Zhang JY, Xie X. COVID-19 and the cardiovascular system. Nat Rev Cardiol. 2020;17(5):259-260. 